\title{
VALIDAÇÃO DA ASSIMILAÇÃO DE DADOS NA INFERÊNCIA DA REFLETIVIDADE DE UM RADAR COM O SISTEMA MM5
}

\author{
GUSTAVO LEITE DA SILVA, ALEX SANTOS DA SILVA, YOSHIHIRO YAMASAKI
}

\author{
Faculdade de Meteorologia, Universidade Federal de Pelotas, Pelotas, RS, Brasil \\ gustavoufpel@gmail.com, alex.meteorologia@gmail.com,yamasaki@fis.ua.pt
}

Recebido Setembro de 2009 - Aceito Maio de 2011

\begin{abstract}
RESUMO
Em certas ocasiões o Estado do Rio Grande do Sul é assolado por sistemas meteorológico com manifestações atmosféricas atípicas e que se enquadram entre os fenômenos de mesoescala. A impossibilidade dos modelos globais simularem adequadamente diversos efeitos localizados, que ocorrem nas mais variadas regiões do globo, associada ao rápido aumento dos recursos computacionais, vem facilitando e induzindo, cada vez mais, o emprego de modelos de mesoescala para melhorar os conhecimentos sobre eventos anômalos e severos e até mesmo para utilização como ferramenta operacional em alguns centros de previsão do tempo. Para se avaliar a destreza de um modelo de mesoescala em prover previsões de precipitação, em escala espacial e temporal compatível com àquelas de um radar meteorológico Doppler, é implantado todo o sistema de modelagem de mesoescala, que constitui o MM5 na exploração da ocorrência de um evento relativamente severo de precipitação, ocorrido na região próxima da cidade de Canguçu/RS, no dia 11 de janeiro de 2008 . O evento foi arbitrariamente selecionado para avaliação do modelo em inferir a refletividade do radar meteorológico instalado na cidade de Canguçu/RS, processando-se com e sem a assimilação de dados convencionais; bem como para abordar a análise da situação termo-hidrodinâmica e sinótica.
\end{abstract}

Palavras Chave: PCA, PSA, refletividade, sistema MM5.

\section{ABSTRACT: VALIDATION OF DATA ASSIMILATION IN THE INFERENCE OF A RADAR REFLECTIVITY WITH THE MM5 SYSTEM}

On certain occasions the Rio Grande do Sul State is affected by atypical weather systems events which are in the category of mesoscale phenomena. The inability of global forecast models to appropriately simulate several localized effects, which occurs over the most different regions, associated to the fast increase of computational resource, is facilitating and increasingly inducing the use of mesoscale models to improve the understanding of anomalous and severe events and also as a necessary and indispensable operational tool. In order to evaluate the skill of mesoscale model to provide precipitation forecasts, with space and time scales compatible with those of a Doppler meteorological radar, a mesoscale modeling system, the MM5, is implemented to explore the occurrence of the relatively severe precipitation event; occurred near the city of Canguçu / RS, on January 11, 2008. The event was arbitrarily selected; to evaluate the model skill in inferring the reflectivity of the meteorological radar, installed in Canguçu / RS, running the MM5 model with and without conventional data assimilations, as well as to get an approach of the thermo-hydrodynamics and synoptic conditions analysis.

Keywords: PCA, PSA, reflectivity, MM5 system.

\section{INTRODUÇÃO}

Devido à inerente natureza localizada de alguns tipos de eventos meteorológicos de mesoescala, eles podem induzir tempestades anomalamente severas (TAS). Esses eventos vêm apresentando grandes desafios à meteorologia, na capacidade de prover a previsão detalhada, com antecedência suficiente que possibilite a tomada de medidas preventivas contra os eventuais danos econômicos e sociais. Alguns autores como Doviak e Zrnic (1993), Wilson e Mueller (1993), Handwerker (2002) mostraram que, em alguns momentos do ciclo de desenvolvimento dos sistemas de mesoescala, que produzem a TAS, a precipitação 
provocada por essas tempestades podem ser detectadas por radares meteorológicos, bem como, para se proceder a avaliação das evoluções das estruturas tanto espaciais como temporais.

A evolução dos radares meteorológicos Doppler tem disponibilizado observações da atmosfera com alta resolução temporal e espacial, e vem se tornando imprescindível à análise e compreensão da dinâmica de fenômenos meteorológicos de mesoescala, bem como, condição inicial e ferramenta comparativa para modelos numéricos de previsão de tempo. Apesar dessa evolução, os radares são ainda pouco utilizados nas práticas operacionais por apresentarem coberturas espaciais restritas, além do elevado custo operacional.

Há inúmeros modelos de mesoescala atualmente disponíveis, a domínio público, dentro da comunidade meteorológica. O sistema MM5 (Dudhia, et al., 2005) é, em particular, o que foi e que ainda vem sendo mais utilizado a nível mundial, tanto para fins científicos como técnicos e até mesmo operacionais, embora alguns centros venham migrando para o sistema WRF. Para se proceder a avaliação da destreza de um modelo de mesoescala em prover previsões de precipitação, na forma de refletividade, em escala espacial e temporal compatível com aquelas de um radar meteorológico Doppler, é implantado o sistema MM5. A ocorrência do evento relativamente severo de precipitação, ocorrido nas proximidades da cidade de Canguçu/ RS, do dia 11 de janeiro de 2008 foi arbitrariamente selecionada, para a avaliação do modelo com e sem a assimilação de dados convencionais, bem como, para proceder à abordagem da análise da situação termo-hidrodinâmica e sinótica.

\section{MATERIAL E MÉTODOS}

O foco principal é envolvido pela região do Estado do Rio Grande do Sul e suas vizinhanças, delimitada entre as latitudes $26^{\circ} \mathrm{S} 46^{\prime} 12^{\prime \prime}$ e $35^{\circ} \mathrm{S} 10^{\prime} 48^{\prime \prime}$ e longitudes $47^{\circ} \mathrm{W} 05^{\prime} 24^{\prime \prime}$ e $60^{\circ} \mathrm{W} 48^{\prime} 00^{\prime \prime}$ (Figura 1).

Os dados que serviram de suporte às análises do evento são: Temperatura do ar, temperatura do ponto de orvalho, pressão atmosférica, direção e velocidade do vento de 70 estações sinóticas de superfície, de 6 em 6 horas, do dia 11 de janeiro de 2008, foram obtidas na http://www.atmos.e. As estações de radiossondagem, com dados das 0000 e 1200 UTC, obtidos no http://weather.uwyo.edu, são apresentadas, com as respectivas altitudes, longitudes, latitudes e número OMM na Tabela 1.

Análise do modelo espectral Global Forecasting System (GFS) do National Center for Environmental Prediction (NCEP) utilizados para a inicialização e de contorno na integração do modelo foram obtidos no http://dss.ucar.edu/datasets/ds083.2.

Informações do radar Doppler de Canguçu, no formato CAPPI, dos 15 níveis de altura, de $2 \mathrm{~km}$ a $16 \mathrm{~km}$, e resolução horizontal de $3 \mathrm{~km}$ por $3 \mathrm{~km}$, processados pelo DAS/INPE.

Imagens do canal infravermelho do satélite GOES-12, do dia 11 de janeiro de 2008, armazenadas no http://www.sat. dundee.ac.uk.

\subsection{Sistema de modelagem MM5}

\subsubsection{Assimilação de dados}

Uma forma de aumentar o realismo das informações, que são, muitas vezes ou até mesmo quase sempre, coletadas e observadas em diferentes locais ou não dos pontos gradeados de um modelo, é dispor de informações sobre os erros das medições de suporte, normalmente designados como erro background, e modificados de forma que dependa do escoamento, pelas equações dinâmicas do modelo, durante o período de assimilação.

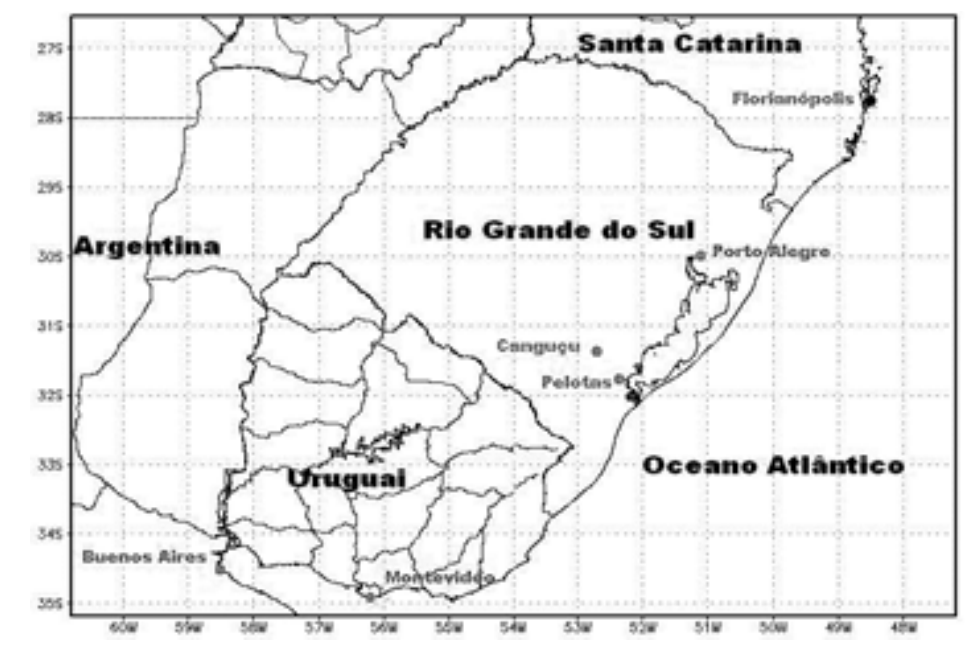

Figura 1 - Região de estudo. 
Tabela 1 - Identificação das estações de ar superior.

\begin{tabular}{c|c|c|c|c}
\hline Estação & WMO & Latitude (graus) & Longitude (graus) & Altitude (metros) \\
\hline Porto Alegre (POA) & 83971 & -30.00 & -51.18 & 03 \\
\hline Santa Maria (RIA) & 83937 & -29.70 & -53.70 & 85 \\
\hline Uruguaiana (URG) & 83928 & -29.78 & -57.03 & 74 \\
\hline Florianópolis (FLN) & 83899 & -27.67 & -48.55 & 05 \\
\hline Resistência (RES) & 87155 & -27.45 & -59.05 & 53 \\
\hline Buenos Aires (EZE) & 87576 & -34.82 & -58.53 & 20 \\
\hline
\end{tabular}

O sistema de assimilação de dados consiste basicamente na combinação de todas as informações disponíveis sobre o estado da atmosfera em uma determinada janela de tempo. Ele produz uma estimativa sobre as condições atmosféricas, válidas para um determinado instante de tempo referido como de análise. As informações empregadas para produzir essa análise incluem, tanto as observações, como as previsões anteriores, que comumente são referidas como o estado de suporte ou de primeira aproximação, assim como os seus respectivos erros e as leis das físicas.

As análises, sobre o aspecto bem amplo, podem ser empregadas de distintas formas na meteorologia. Elas podem prover as condições iniciais, ou seja, de inicialização para os modelos de previsões de tempo. Da mesma forma, podem também ser empregadas para se proceder as pesquisas climáticas pela re-análise, com a inclusão de observações e resultados de modelos numéricos. Dentre muitos outros, podem ainda ser utilizadas para prever o potencial, bem como, avaliar e simular o impacto dos componentes individuais de uma rede de observações em um sistema de previsão de tempo.

O sucesso do processamento do modelo, com assimilação de dados (PCA) e previsão numérica do tempo, depende crucialmente da precisão com que as condições iniciais são fornecidas ao mesmo. A relativa importância dos erros das previsões, que se devem às condições iniciais, comparada com as de outras fontes de erros, tais como aquelas devidas às parametrizações físicas, condições de contorno e previsões dinâmicas, dependem de inúmeros fatores, como a da resolução espacial e temporal, áreas dos domínios das integrações, densidade de dados disponíveis, orografias, além do próprio produto da previsão propriamente dito.

As aplicações iniciais do sistema 3DVAR no MM5 têm sido focadas, para prover as condições iniciais, para a integração das previsões do MM5. Despende-se grande esforço no desenvolvimento do sistema de assimilação variacional de dados para substituir o esquema, por exemplo, o de Cressman (MM5) e o de "nudging" Newtoniano (FDDA - MM5).

Considerações práticas têm levado a uma variedade de implementações alternativas do sistema VAR. O principal objetivo do sistema básico 3DVAR do MM5 é produzir uma estimativa ótima do estado real da atmosfera no instante da análise, através da solução iterativa de uma função custo prescrita (Ide et al., 1997).

$$
J(x)=J^{b}+J^{o}=\frac{1}{2}\left(x-x^{b}\right)^{T} B^{-1}\left(x-x^{b}\right)+\frac{1}{2}\left(y-y^{b}\right)^{T}(E-F)^{-1}\left(y-y^{o}\right)
$$

O problema do VAR resume-se na solução iterativa da Equação 1. Ele consiste na obtenção do estado x que minimiza a função custo $\mathrm{J}(\mathrm{x})$. A solução representa desta forma, o valor final do máximo da possibilidade (ou seja, com o mínimo de variância) estimada para o estado verdadeiro da atmosfera, ao fornecer duas fontes de dados a priori: o de suporte, tomado como sendo aquela do prognóstico anterior $\mathrm{x}^{\mathrm{b}} \mathrm{e}$ as observações $\mathrm{y}^{0}$ (Lorenc, 1986). Para se ajustar aos pontos de dados individuais da grade, os valores são ponderados pelas estimativas de seus erros: B, E e F, que são as covariâncias, respectivamente das matrizes de suporte, da observacional (instrumental) e do erro representativo. A função custo quadrática, dada pela Equação 1, supõe que as covariâncias dos erros das observações e dos suportes são estatisticamente descritos por funções Gaussianas de densidade de probabilidade, com erro médio nulo. Alternativamente as funções custo podem ser usadas para relaxar essas suposições, conforme apresentado por (Dharssi et al., 1992). O emprego das operações adjuntas, que pode ser vista como uma aplicação multidimensional da regra em cadeia - para a diferenciação parcial permite calcular, de forma eficiente, o gradiente da função custo. O problema teórico da minimização da função custo $\mathrm{J}(\mathrm{x})$ é equivalente à da geração anterior, da técnica da interpolação ótima (OI) do caso linear. 


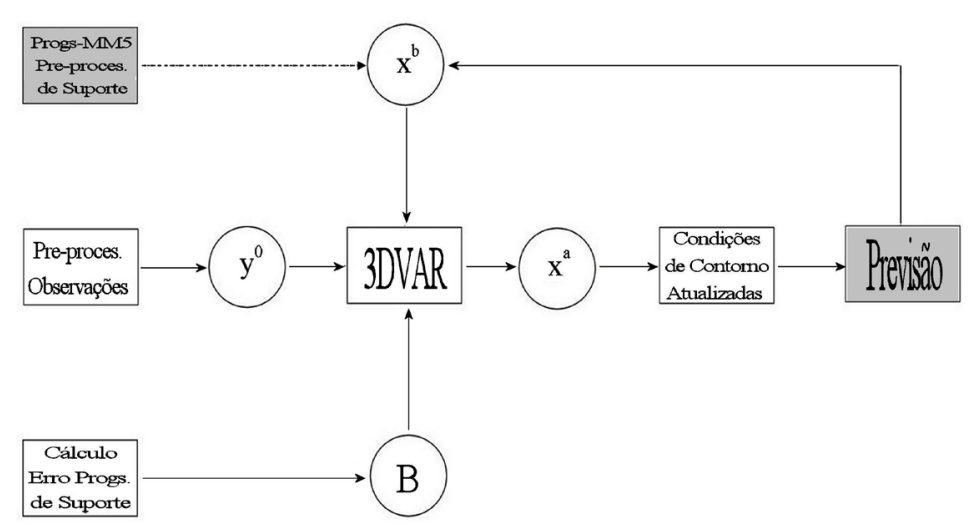

Figura 2 - Visão geral do sistema 3DVAR usado no MM5.

Na Figura 2 é apresentada uma visão geral do sistema 3DVAR usado no MM5. A configuração básica apresenta o modo comumente referido como "cold start', onde a previsão de suporte é resultante do produto de algum outro modelo, tais como, os prognósticos dos modelos globais da atmosfera, como o do GFS/NCEP, ECNWF, CPTEC, etc. O outro modo é o cíclico, em que a previsão de suporte é a da própria previsão de curto prazo do MM5, produzida com o processamento da análise com 3DVAR anterior. As três formas de arquivos de entrada de dados, o first guess, observação e erro de suporte, e as saídas (análises) são apresentadas em círculos. Os retângulos não hachureados apresentam códigos fontes destinados a serem usados com o 3DVAR e o MM5, os demais representam os códigos pré-existentes no MM5 sem o 3DVAR. No primeiro modo o processamento é o padrão normal, que se processa mesmo sem a assimilação de dados. No modelo cíclico, o processamento de suporte não é necessário, pois o campo de suporte $\mathrm{x}^{\mathrm{b}}$ para o 3DVAR já está na própria grade do MM5.

$O$ pré-processador fornece as observações $\mathrm{y}^{0}$ para serem ingeridas pelo 3DVAR. O programa 3DVAR_OBSPROC apresenta distintas funções básicas. Ele lê os dados das observações em um formato especial conhecido como formato MM5 LITTLE-R (Dudhia, et al., 2005), faz a verificação espacial e temporal dos dados observados, obtém as alturas, das observações em coordenadas de pressão, une os dados duplicados e seleciona as observações que apresentam diferentes valores em distintos tempos, além de estimar os erros de cada observação e produzir dados no formato adequado para o processamento do MM5.

As estatísticas das covariâncias de erros de suporte são empregadas na função custo do 3DVAR para ponderar os erros relacionados com os campos de suporte. O sistema de assimilação filtra as estruturas de suporte e de observação, que apresentam altos erros relativos àquele mais corretamente conhecidos. Na realidade os erros dos campos de suporte serão sinoticamente dependentes, ou seja, variam de dia para dia dependendo da situação e condição do tempo (Parrish e Derber, 1992).

\subsubsection{Configuração e processamento}

O modelo MM5 foi configurado sobre o domínio que envolve toda a região do Rio Grande do Sul, Uruguai, o sul de Santa Catarina e o nordeste da Argentina (Figura 3). O primeiro módulo do MM5 (TERRAIN) foi configurado com 3 grades aninhadas conforme apresentado na Figura 3. O espaçamento horizontal, entre os pontos de grade do domínio maior (D1) foi estabelecido em $27 \mathrm{~km}$, no sub-domínio intermediário (D2) foi de $9 \mathrm{~km}$ e o domínio menor (D3) foi de $3 \mathrm{~km}$. Os dois maiores domínios do sistema foram centrados na latitude de $31^{\circ} \mathrm{S}$ e longitude $54^{\circ} \mathrm{W}$, e, o domínio menor foi centralizado na latitude e longitude de instalação do radar meteorológico de Canguçu, localizado em $31^{\circ} 24^{\prime} 14^{\prime \prime}$ S e $52^{\circ} 42^{\prime} 06^{\prime \prime}$ W.

O REGRID é o segundo módulo do sistema MM5 e foi processado para condicionar os campos meteorológicos de acordo com as grades dos domínios de integração. Ele foi configurado para o processamento durante o período compreendido entre as 0000 UTC do dia 11/01/08 até às 0000 UTC do dia 12/01/08, utilizando previsões do modelo GFS. O módulo LITTLE_R, que interpola espacial e temporalmente, bem como, dispõe os dados de estações convencionais nos pontos de grade de cada um dos domínios de integração do modelo foi processado para cada um dos domínios de integração. Nesse módulo foram incorporados os dados das observações convencionais de superfície e altitude para proceder ao PCA. Os dados foram também preparados para o processamento do caso sem assimilação de dados (PSA). O programa INTERPF foi posteriormente processado para interpolar os campos meteorológicos, dos níveis de pressão produzidos pelo módulo LITTLE_R, para níveis sigma, que é a coordenada vertical do modelo MM5. Esse módulo não apenas perfaz a interpolação vertical, como também proporciona os diagnósticos computacionais e a re-formatação dos dados para 


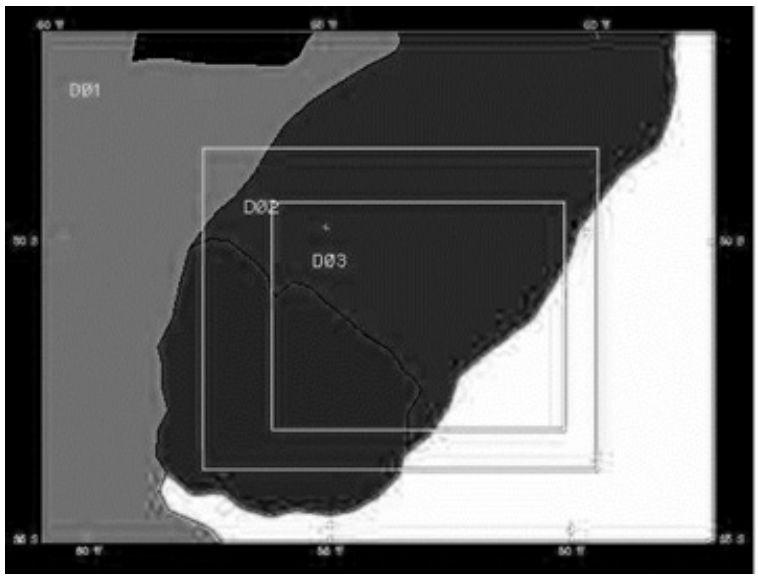

Figura 3 - Domínios D01 (27 km), D02 (9 km) e D03 (3 km).

o modelo MM5. Tendo em vista que o interesse principal está condicionado, principalmente nos baixos níveis da atmosfera, foram selecionados 26 níveis sigma na vertical, com grande resolução vertical próximos à superfície.

O módulo MM5 que perfaz a previsão numérica do tempo do sistema de modelagem propriamente dita, foi configurado com parametrizações da microfísica, convecção, camada limite planetária, radiação e de temperatura de solo. Na Tabela 2 são apresentadas as parametrizações estabelecidas, para cada domínio de integração do modelo. $\mathrm{O}$ modelo foi processado para o período de 24 horas, a partir das $0000 \mathrm{~h}$ do dia 11/01/2008, provendo previsões, devidamente armazenados, com intervalos de 30 minutos.

\subsection{Radar meteorológico}

O radar meteorológico está instalado na cidade de Canguçu (RS), a 466,57 metros de altitude, localizado na latitude $31^{\circ} \mathrm{S} 24^{\prime} 14^{\prime \prime}$ e longitude $52^{\circ} \mathrm{W} 42^{\prime} 06^{\prime \prime}$ e é operado pelo Departamento de Controle do Espaço Aéreo (DECEA). Trata-se de um radar Doppler que opera em banda S (2,7 - 3

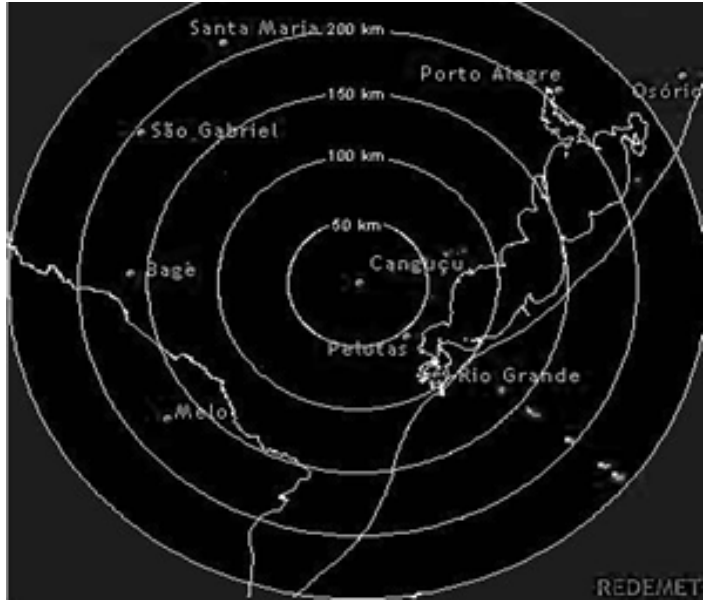

Figura 4 - Área de cobertura do radar de Canguçu/RS. (Fonte: http// www.redemet.aer.mil.br/radar - adaptado)

$\mathrm{GHz}$ ), com comprimento de onda de $10 \mathrm{~cm}$ e apresenta uma abertura do feixe de microondas de aproximadamente $2^{\circ}$. Ele leva 15 minutos para executar 15 varreduras volumétricas, e é operado por 24 horas por dia. Na Figura 4 é apresentada a área de cobertura do radar de Canguçu, para um raio de 250 $\mathrm{km}$. Entretanto, para todo o mês de janeiro de 2008, o DECEA disponibilizou apenas os dados com alcance máximo delimitado em $69 \mathrm{~km}$, para todos os ângulos de elevações.

O Instituto Nacional de Pesquisas Espaciais (INPE), por um convênio estabelecido com o DECEA, acessa os dados brutos do radar de Canguçu e perfaz o processamento dos mesmos. No processamento eles são colocados no formato CAPPI ("Constant Altitude Plan Position Indicator") e formatados em 15 níveis verticais, de $2 \mathrm{~km}$ a $16 \mathrm{~km}$. O produto CAPPI é obtido a partir da refletividade do radar, coletados de cada azimute e elevação. No final do processo da varredura eletrônica, o volume tridimensional de dados do radar é o que possibilita obter todo o perfil vertical da refletividade do radar em $\mathrm{dBz}$, dentro da área de cobertura. O CAPPI é obtido com os dados da varredura completa do radar, em todas as

Tabela 2 - Parametrizações da microfísica, convecção, camada limite planetária, radiação e temperatura do solo.

\begin{tabular}{c|c|c|c}
\cline { 3 - 4 } & \multicolumn{3}{c}{ Parametrizações } \\
\cline { 2 - 4 } & (D1) $27 \mathrm{~km}$ & (D2) $9 \mathrm{~km}$ & (D3) 3km \\
\hline Microfísica & Schultz & Schultz & Graupel (reisner2) \\
\hline Convecção & Kuo & Kain-Fritsch 2 & Explícita \\
\hline Radiação & Dudhia & Dudhia & Dudhia \\
\hline Solo & 5 camadas & 5 camadas & 5 camadas
\end{tabular}




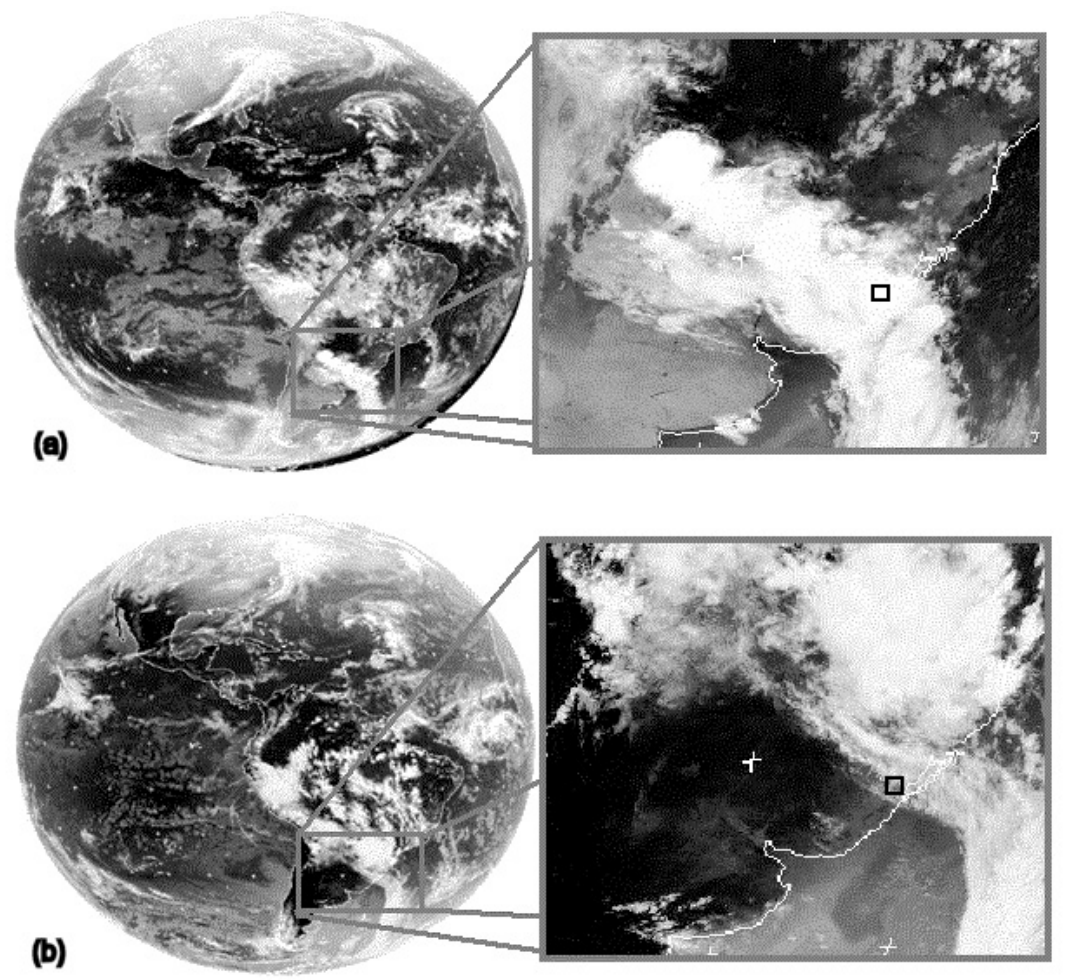

Figura 5 - Imagem do canal infravermelho do satélite GOES-12, do dia 11/01/2008 das (a) 0600UTC e (b) 2100 UTC.

elevações da antena, na altura desejada. Após fixar a altura, é feita a interpolação das informações, contidas em cada uma das elevações da PPI ("Plan Position Indicator"), formando assim um campo de refletividade em um plano de altitude constante.

Como a resolução horizontal dos dados do radar $(1 \mathrm{~km}$ por $1 \mathrm{~km}$ ) é maior que a do terceiro domínio do modelo (3 $\mathrm{km}$ por $3 \mathrm{~km}$ ), eles foram devidamente processados para apresentação compatível com a do modelo.

\section{RESULTADOS E DISCUSSÃO}

No dia 11 de janeiro de 2008 ocorreu a entrada de uma frente fria sobre o Rio Grande do Sul, combinado com a presença de uma massa de ar quente e úmida que atuava sobre o Estado. Esse sistema provocou ventos fortes, pancadas de chuva e descargas elétricas em várias localidades. De acordo com informações do Instituto Nacional de Meteorologia (INMET), foram registradas, entre as $10 \mathrm{~h}$ do dia 10 e $10 \mathrm{~h}$ do dia 11 , precipitação acumulada de 21,2mm em Porto Alegre; 29,4mm em Encruzilhada do Sul e 76,5mm em Rio Grande.

A imagem, do canal infravermelho, do satélite GOES12 das 0600UTC, do dia 11/01/2008 (Figura 5a), apresenta claramente uma frente fria caracterizada por uma banda de nuvens espessas, em forma de vírgula, que se estende desde o Nordeste da Argentina até o Atlântico Sul. Também é possível observar, mais ao Sul do Oceano Atlântico, uma circulação, no sentido horário, associada a um ciclone extratropical. $\mathrm{Na}$ imagem, das 2100UTC (Figura 5b), é nítida a presença do sistema convectivo que se desenvolveu durante o dia, atuando em todo Estado do Rio Grande do Sul. Posteriormente, com a oclusão do sistema sobre o Oceano, a frente fria perdeu sua intensidade e se afastou do continente. O retângulo, apresentado em ambas as figuras, representa uma ampliação da área de interesse, onde o quadrado preto representa a área de cobertura do radar, localizado na cidade de Canguçu.

Na Figura 6 é apresentada uma seqüência, de 3 em 3 horas, de imagens de alta resolução, do canal infravermelho do satélite GOES-12, das 0300UTC às 1200UTC do dia 11/01/2008. O ponto preto, situado no Estado do Rio Grande do Sul, representa a cidade de Canguçu. A análise da seqüência de imagens apresentadas revela claramente a ocorrência do avanço da frente fria, proveniente do Sul do Uruguai. Esse sistema frontal se desenvolve ao avançar em direção do Rio Grande do Sul e chega até a fronteira entre o Brasil e o Uruguai. Às 0600UTC a frente fria já se destaca na região Sul e Oeste do Estado do Rio Grande do Sul (Figura 6b). Tanto na imagem das 0600UTC, como na das 0900UTC (Figura 6c), é possível notar que a nebulosidade causada pelo sistema frontal em superfície, atingiu níveis mais elevados até então, com uma visível formação de aglomerados de nuvens do tipo $\mathrm{Cb}$, que 



Figura 6 - Imagens de alta resolução, do canal infravermelho do satélite GOES-12, para as 0300UTC as 1200UTC, a cada 3horas.

são nuvens de grande desenvolvimento vertical, que provocam intensa precipitação, fortes rajadas de vento e descargas elétricas, principalmente na região Noroeste do Rio Grande do Sul e Nordeste da Argentina. O deslocamento da frente fria, do continente em direção ao Oceano Atlântico, é percebido no instante seguinte, onde pode-se perceber a configuração de um Sistema Convectivo de Mesoescala (SCM) que se desenvolveu na região do Nordeste da Argentina e Noroeste do Rio Grande do Sul.

Nas Figuras 7 e 8 são apresentados os campos simulados de água precipitável, vento a $10 \mathrm{~m}$ e temperatura a $2 \mathrm{~m}$, para o Processamento Com Assimilação de dados (PCA) (a) e Processamento sem assimilação de dados (PSA) (c) e as imagens do canal infravermelho do satélite GOES-12 (b), das 0300UTC e às 0600UTC. Na Figura 7 é possível observar que no horário das 0300UTC, os dois processamentos mostram-se condizentes com a imagem de satélite. Essa imagem mostra o avanço da frente fria pelo Uruguai em direção ao Rio Grande do Sul e por conseqüência, a chegada desse sistema na fronteira entre Brasil e Uruguai. As linhas isotérmicas dos dois processamentos conseguem simular a frente fria, situada na região onde o gradiente de temperatura é mais forte. A inversão no sentido do vento para a direção Norte, próximo a superfície, com a passagem do sistema frontal, também pode ser observada nos dois processos. Comparando nebulosidade da imagem do satélite com o campo de água precipitável simulada, pode-se perceber que as áreas de maior quantidade de água precipitável correspondem às áreas de maior nebulosidade. No PCA e PSA as áreas de maior quantidade de água precipitável correspondem a região onde está localizada a frente fria, sendo que o PSA mostra uma pequena região com valores máximos de água precipitável (acima de $55 \mathrm{~mm}$ ) no Nordeste da Argentina, que o PCA não apresenta.

Com o avanço do sistema frontal, a nebulosidade chega ao Rio Grande do Sul deixando o céu encoberto em todo o sul do Estado (Figura 8b). Os dois processamentos são bem semelhantes e conseguem mostrar o avanço da frente fria sobre o Rio Grande do Sul, mostrando que as regiões com maior nebulosidade correspondem às regiões de maior quantidade de água precipitável. Nesse instante, o PCA apresenta um núcleo bem definido de intensos valores de água precipitável no Oeste do Estado gaúcho (acima de $55 \mathrm{~mm}$ ), diferentemente do PSA que não mostra esse núcleo.

Comprovada a lisura do modelo, em prover simulações da atmosfera, necessita-se de um sistema para comparar a refletividade simulada com os dados observados por radar. Os dados da refletividade do radar estão em formato CAPPI; ou seja, esses dados estão dispostos em alturas constantes em relação ao radar. Sabe-se que o radar está localizado a uma altura de 466m acima da superfície do mar, e que o modelo de previsão numérica MM5 é configurado em coordenadas verticais sigma $(\sigma)$ que, portanto, acompanha o contorno do relevo. Na Figura 9 é apresentado, como exemplo, os efeitos da topografia nas superfícies que podem ser inferidos sobre uma 


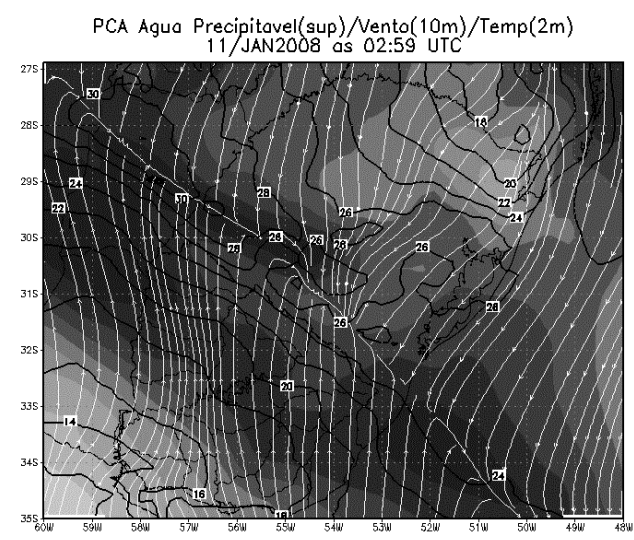

(a) PCA- 03:00UTC

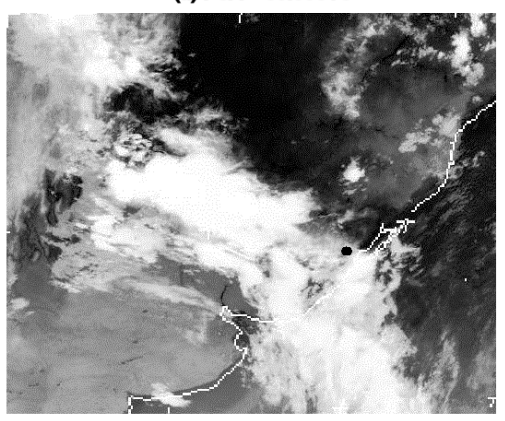

(b) 03:00UTC PSA Aguo Precipitovel'sup)/Vento $(10 \mathrm{~m}) / \operatorname{Temp}(2 \mathrm{~m})$
$11 / \mathrm{JAN} 2008$ as $02: 59$ UTC

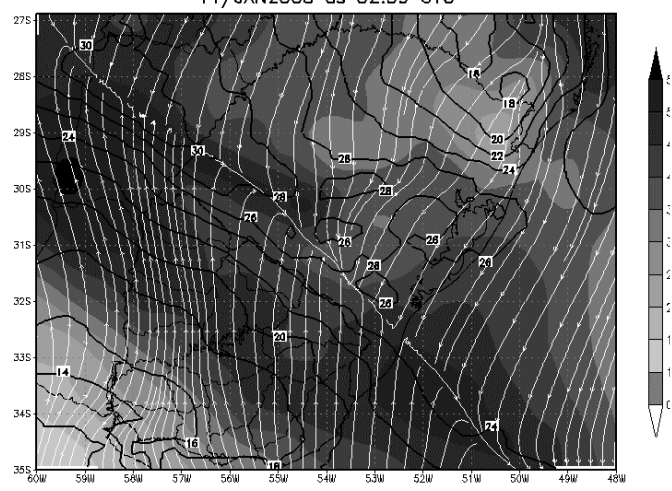

(c) PSA-03:00UTC

Figura 7 - Água precipitável, vento a 10m e temperatura a 2m, PCA (a) e PSA (c) e imagem do canal infravermelho GOES-12 (b), para as 0300UTC.

isóbara. O exemplo mostra que não é somente a pressão que sofre a influência dos efeitos da topografia. A temperatura, o vento e a umidade, por exemplo, também tendem a seguir um comportamento similar na proporção em que a altitude aumenta, mostrando que para comparar a refletividade simulada com a refletividade observada, muitos fatores devem ser levados em consideração. O erro cometido na comparação entre a refletividade simulada com a observada, na relação altura e pressão, é inversamente proporcional a altitude. Entretanto, o fato é que em altos níveis da atmosfera há um

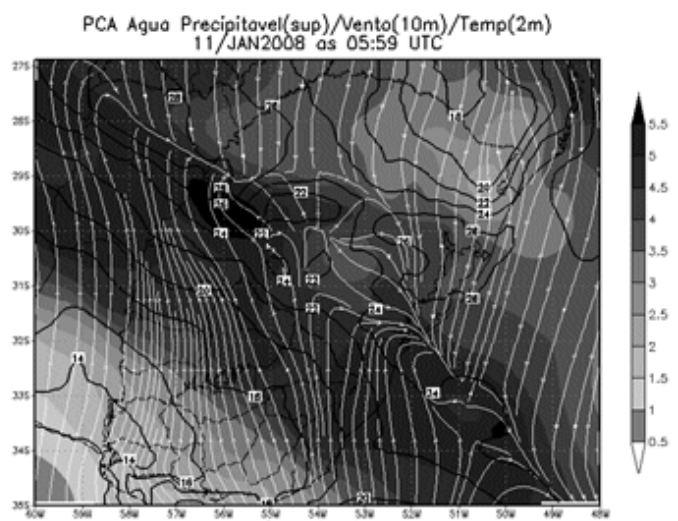

(c) PSA-06:00UTC

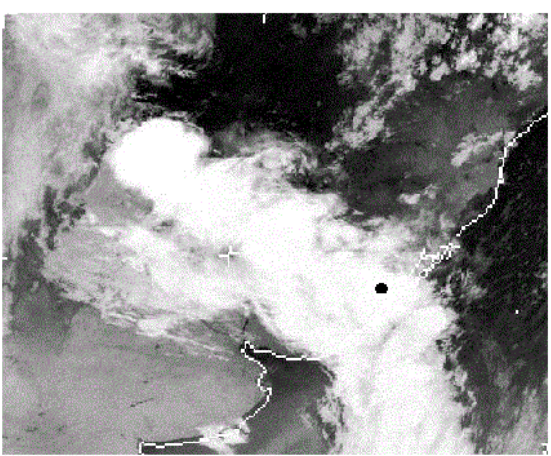

(a) 06:00UTC

PSA Aguo Precipitovel(sup)/ Nento(10m)/Temp(2m)

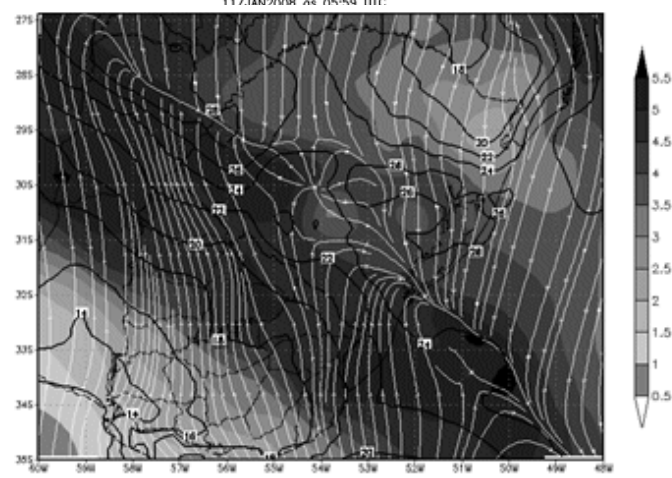

(A) Ra-caecuro

Figura 8 - Água precipitável, vento a $10 \mathrm{~m}$ e temperatura a $2 \mathrm{~m}$, PCA (a) e PSA (c) e imagem do canal infravermelho GOES-12 (b), para as 0600UTC.

aumento na depressão, ou seja, na diferença entre a temperatura e a temperatura do ponto de orvalho, o que implica em uma diminuição expressiva da umidade nos níveis mais elevados. Então, não adianta reduzir o erro, meramente adotando um nível de pressão em altitudes elevadas se a atmosfera estiver seca, pois, em uma atmosfera com baixa umidade não há formação de nuvens e por conseqüência não haverá partículas de chuva.

Nas Figuras 10 e 11 são apresentadas a refletividade simulada com PCA (a) e PSA (c) em $600 \mathrm{hPa}$ e a refletividade 


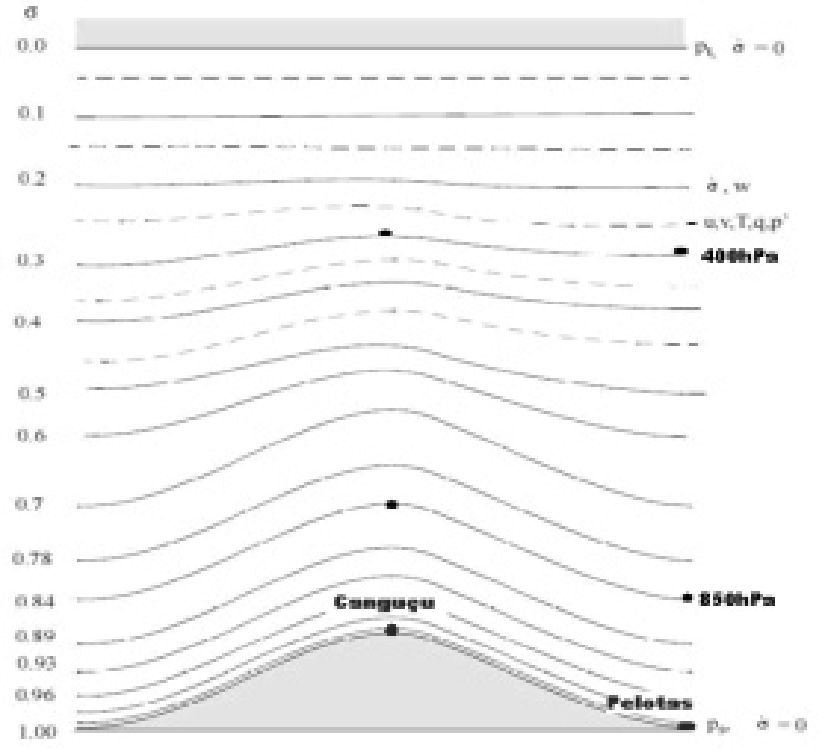

Figura 9 - Efeitos da superfície na pressão, temperatura, umidade e vento. Fonte: http://www.redemet.aer.mil.br/mm5 (adaptado)

observada no nível de $4 \mathrm{~km}$ (b), para os horários das 0600UTC e 1200UTC, respectivamente. Nas figuras da refletividade observada pelo radar, é possível notar que às 0600UTC (Figura 10b) o radar começa a registrar valores significativos de refletividade (da ordem de 40, 45 e 50dBz), avançando do setor oeste/sudoeste em direção ao da localização do radar. O período compreendido entre 0600UTC a 1200UTC, nas figuras observadas, corresponde àquele em que se constata a maior quantidade de partículas e valores mais elevados de refletividade. Nas imagens de satélite, para os mesmos horários correspondentes, o intervalo de maior nebulosidade sobre a área observada, também corresponde aos mesmos horários com valores mais elevados de refletividade observada pelo radar, evidenciando não apenas a relação entre a refletividade e a nebulosidade, como também, mostrando que os dados apresentam coerentes com a observação.

A comparação entre a refletividade observada e as simuladas (Figura 10), que se refere ao instante em que são observados valores significativos da refletividade no setor oeste/sudoeste da área de alcance do radar, mostra que os dois processamentos (PCA e PSA) não registraram as refletividades apresentadas pelo radar meteorológico. O PCA apresenta algumas refletividades com valores variando de 20 a $40 \mathrm{dBz}$ a leste do radar; o que é também apresentado pelo PSA; mas, com valores inferiores e locais igualmente distintos.

A Figura11b, às 1200UTC, mostra que a observação apresenta os maiores valores de refletividade, próximos aos extremos da área do alcance do radar, e valores mínimos e medianos espalhados por todas as demais áreas. Os dois

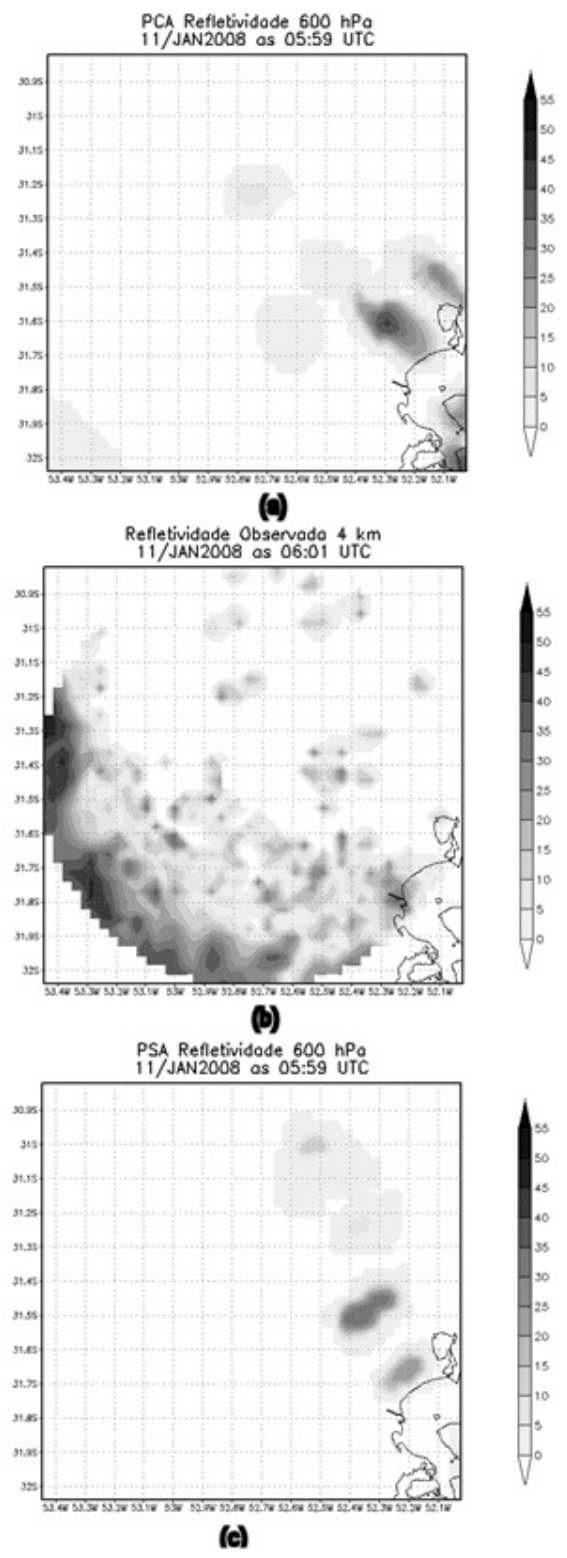

Figura 10 - Refletividade simulada com PCA (a) e PSA(c) em $600 \mathrm{hPa}$ e a refletividade observada no nível de $4 \mathrm{~km}$ (b), para as 0600UTC.

processamentos (PSA e PCA) não apresentaram os valores extremos nos setores observados e, em praticamente toda área, subestimam os valores das refletividades observadas, exceto nos locais com valores elevados da refletividade.

\section{CONCLUSÕES}

O sistema MM5 foi configurado com três domínios aninhados de integração, de forma que aquele com maior resolução espacial (D3) apresenta uma resolução espacial de 3 $\mathrm{km}$, e inclui todo o range de cobertura do radar meteorológico Doppler, com alcance máximo estabelecido em $69 \mathrm{~km}$, para fins 


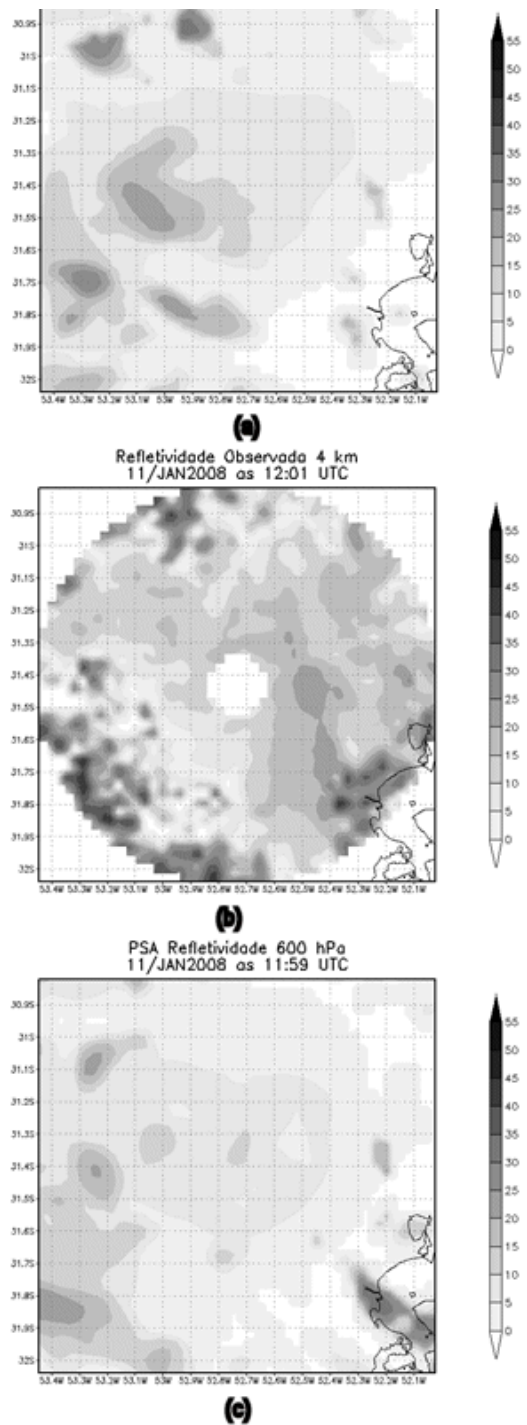

Figura 11 - Refletividade simulada com PCA(a) e PSA (c) em 600hPa e a refletividade observada no nível de $4 \mathrm{~km}$ (b), para as $1200 \mathrm{UTC}$.

de comparação da refletividade simulada com a refletividade observada nos dados do radar meteorológico de Canguçu/RS.

As diferenças das refletividades, entre os processamentos PCA e PSA são bastante evidentes em todos os processos empregados na comparação dos resultados; mas nenhum deles apresentou uma real e inequívoca eficiência em simular a refletividade do radar. Entretanto, na comparação entre as refletividades simuladas, é visível a grande diferença entre os dois processos, mostrando que o fato de processar o modelo com dados de estações locais e com alta resolução é um fator relevante para a refletividade.

As análises desenvolvidas revelaram que o modelo de mesoescala MM5 permite prognosticar e proporcionar previsões que possibilitam a inferência das refletividades de um radar meteorológico, sobretudo quando da assimilação de dados convencionais.

\section{AGRADECIMENTOS}

Às instituições, INPE, NCEP, DECEA,DUNDEE e NCAR, pela disponibilização dos dados. À CAPES pela concessão da bolsa de mestrado e à Universidade Federal de Pelotas pelo apoio à realização dessa pesquisa.

\section{REFERÊNCIAS BIBLIOGRÁFICAS}

DHARSSI, I.; LOREN, A. C.; INGLEBY, N. (1992). Treatment of gross errors using maximum probability theory. Quaterly Journal of the Royal Meteorological Society, 118:10171036.

DOVIAK, R. J.; ZRNIC, D. S. Doppler radar and weather observations. Academic Press, Inc., San Diego, 458pp. 1993.

DUDHIA, J.; GILL, D.; MANNING,K.; WANG, W.; BRUYERE, C. PSU/NCAR Mesoscale Modeling System Tutorial Class Notes and User's Guide (MM5 Modeling System Version 3.7). Mesoscale and Microscale Meteorology Division. National Center for Atmospheric Research. 2005.

HANDWERKER, J. Cell tracking with TRACE3D - a new algorithm. Atmospheric Research, v.61, n.1, p.15-34, 2002.

IDE, K., P. COURTIER, M. GHIL; A. LORENC, 1997: Unified notation for data assimilation: Operational, sequential and variational. J. Meteor. Soc. Japan, 75, 181-189.

LORENC, A. C., 1986: Analysis methods for numerical weather prediction. Q. J. R. Meteorol. Soc., 112, 1177-1194.

PARRISH, D. F.; J. DERBER, 1992: The National Meteorological Center's spectral statistical Interpolation analysis system. Mon. Wea. Rev., 120, 1747-1763.

WILSON, J. W.; MUELLER, C.K. Nowcasts of Thunderstorm Initiation and Evolution. Weather Forecasting, v.8, n.1, p.113-131, 1993. 